\title{
RESONANT PRODUCTION OF SOLITONS IN THE RLW EQUATION
}

\author{
J. COURTENAY LEWIS ${ }^{1}$ and J.A. TJON \\ Institute for Theoretical Physics, University of Utrecht, 3508 TA Utrecht, The Netherlands
}

Received 31 July 1979

\begin{abstract}
It is found that soliton collisions in the RLW equation can produce numerous pairs of positive- and negative-amplitude solitons. If the initial solitons are parametrized by their areas, the inelasticity of their collision has a sharp maximum along a line which is close to the line of equal but opposite areas.
\end{abstract}

It is well known that soliton-soliton collisions in the Korteweg-de Vries equation are perfectly elastic in the sense that the solitons present after a collision are equal in number, amplitude, and velocity to those present before the collision. However, collisions of solitons satisfying other nonlinear wave equations may exhibit a variety of inelastic behaviour [1--5]. In particular, inelastic collisions of solitons in the regularized long wave (RLW) equation have been studied in two fairly recent publications $[3,4]$. In ref. [3] it was found that in an "overtaking" collision, the two solitons were diminished slightly in amplitude while an oscillating wave was produced. Santarelli [4] followed the collision of two solitons moving in opposite directions and observed the formation of an additional pair of solitons.

In this note we show that the amount of inelasticity in soliton-soliton collisions can be so substantial that several pairs of solitons are formed. We have observed the production of as many as seven pairs and there is no reason to believe that that is an upper limit. If the initial areas are sufficiently small, it is also possible for the two solitons to annihilate each other, with no solitons emerging from the collision. The formation of the additional solitons occurs in a restricted region in the parameter space of the original solitons, as does the annihilation.

\footnotetext{
${ }^{1}$ Permanent address: Department of Physics, Memorial University of Newfoundland, St. John's, Newfoundland, A1B $3 \times 7$, Canada.
}

\section{For the RLW equation}

$w_{t}+(1+w) w_{x}-w_{x x t}=0$,

there are three known invariants [9]:

$$
\begin{aligned}
& I_{\mathrm{I}}=\int_{-\infty}^{\infty} w \mathrm{~d} x, \quad I_{\mathrm{II}}=\int_{-\infty}^{\infty}\left(w^{2}+w_{x}^{2}\right) \mathrm{d} x, \\
& I_{\mathrm{III}}=\int_{-\infty}^{\infty}\left(\frac{1}{3} w^{3}-w_{x}^{2}\right) \mathrm{d} x,
\end{aligned}
$$

or linear combinations thereof. In the following we denote by $I_{\mathrm{Ii}}^{(1)}, I_{\mathrm{If}}^{(1)}$ the initial and final values of the first invariant for soliton 1, and similarly for the other invariants and for soliton $2 . I_{\mathrm{Ii}}^{(1)}$ is thus the signed area of soliton 1 before the collision. The soliton solutions of eq. (1) are given by

$w(x, t)=A \operatorname{sech}^{2} p(x-\bar{x}-v(p) t)$,

with

$A=12 p^{2} /\left(1-4 p^{2}\right) \quad$ and $\quad v(p)=1 /\left(1-4 p^{2}\right)$.

For $p<\frac{1}{2}$ the amplitude $A$ is positive and the soliton is rightward moving, while for $p>\frac{1}{2}, A$ is negative and the soliton is leftward moving. We shall henceforth refer to such positive- and negative-amplitude solitons as "p-solitons" and " $n$-solitons", respectively, using the unqualified term "soliton" to refer to either. From eq. (4) it is clear that either $A>0$ or $A<-3$; i.e. there is a forbidden range of amplitudes. 
We used the same numerical technique for the solution of eq. (1) as Santarelli [4]; that is, eq. (1) was approximated by the three-level scheme of Eilbeck and McGuire [6,7], using the two-level scheme of Peregrine, as described in ref. [6], to start it. Most of our calcu. lations were performed with a spatial step size $h=0.1$, and a time step size $\tau=0.05$, whereas Santarelli used $h=0.2$ and $\tau=0.1$. All our calculations were carried out in single precision on a Cyber 175 .

After a collision, the largest p-soliton and n-soliton were followed until they were clearly detached from the collision-produced solitons, if any, and from any oscillatory background. Their amplitudes were then determined from a quadratic interpolation formula fit to three values of $w$ around each maximum, and the parameter $p$ and the three invariants were then obtained from each soliton amplitude via eqs. (3) and (4). This prescription necessitated rather lengthy runs for resonant collisions, for three reasons: (a) the time delay is long for such collisions, (b) the n-solitons are of small amplitude and hence slow-moving, requiring a long time to separate from the oscillatory background, (c) several solitons of approximately equal amplitude, and hence approximately equal velocity, are produced, and these take a long time to separate from each other. Thus in Santarelli's case, for which $p_{1}=0.40$ and $p_{2}=0.60$, the collision is complete by time $t=16$, whereas for the resonant collision with $p_{1}=0.40$ and $p_{2}=0.616$ shown in fig. 4 , the rightward-travelling disturbance has not completely separated into isolated $\mathrm{p}$-solitons even at $t=110$. Although with the step sizes we used, isolated solitons showed negligible amplitude changes over time intervals as great as $\mathbf{2 0}$ or $\mathbf{3 0}$ units (i.e. several hundred time steps), we found that even apparently slight soliton-soliton or soliton-background overlap led to significant errors in amplitude estimation.

There is no obvious single best measure of the inelasticity of a collision. In ref. [3] the ratio of the amplitude of the larger soliton after a collision to its amplitude before the collision is used. We prefer to define inelasticity in terms of the second invariant $I_{\mathrm{II}}$ defined in eq. (2):

$\kappa_{\mathrm{II}} \equiv 1-\left(I_{\mathrm{IIf}}^{(1)}+I_{\mathrm{IIf}}^{(2)}\right) /\left(I_{\mathrm{IIi}}^{(1)}+I_{\mathrm{IIi}}^{(2)}\right)$.

Similar inelasticities $\kappa_{\mathrm{I}}$ and $\kappa_{\mathrm{III}}$ can be defined using the absolute values of the first and third invariants, respectively. Definition (5) is preferred because $I_{I I}$
$=I_{\mathrm{II}}^{(1)}+I_{\mathrm{II}}^{(2)}$ is conserved throughout the collision whereas $\left|I_{\mathrm{Ii}}^{(1)}\right|+\left|I_{\mathrm{Ii}}^{(2)}\right|$ and $\left|I_{\mathrm{IIIi}}^{(1)}\right|+\left|I_{\mathrm{IIIi}}^{(2)}\right|$ are not; hence it is a priori evident that $0 \leqslant \kappa_{I I} \leqslant 1$. Nevertheless, we found that the three measures $\kappa_{I}, \kappa_{\text {II }}$ and $\kappa_{\text {III }}$ showed the same trends: if a given case was less elastic than another according to one of the measures, it was less elastic by the other two measures as well. We found that $0 \leqslant \kappa_{I} \leqslant \kappa_{\text {II }} \leqslant \kappa_{\text {III }} \leqslant 1$, the only apparent exceptions being a few nearly elastic cases where all three measures were small and lay within the range of numerical error of each other. Although we did not systematically calculate and plot the amplitude ratio used in ref. [3] as a measure of the inelasticity, it was evident in the course of data reduction that it tended to follow the same trend as the other measures.

In general we found in our investigations that in cases where additional solitons were formed in a collision, the inelasticity was large. The converse is not necessarily true; thus for $\frac{1}{2}\left(I_{\mathrm{Ii}}^{(1)}-I_{\mathrm{Ii}}^{(2)}\right)=10$, no additional solitons are produced even though $\kappa_{I I}$ can exceed $30 \%$ along this line, or $100 \%$ if the annihilating collisions are included. Whether solitons are produced or not, $\kappa_{I I}$ is sharply peaked for collisions in which the two solitons initially have areas of opposite sign and approximately equal magnitude, i.e. for $I_{\mathrm{Ii}}^{(1)} \approx$ $-I_{\mathrm{Ii}}^{(2)}$. In addition, the larger the area of the initial solitons with $I_{\mathrm{Ii}}^{(1)} \approx-I_{\mathrm{Ii}}^{(2)}$, the more solitons are produced. Fig. 1 shows the production of three p,n-soli-

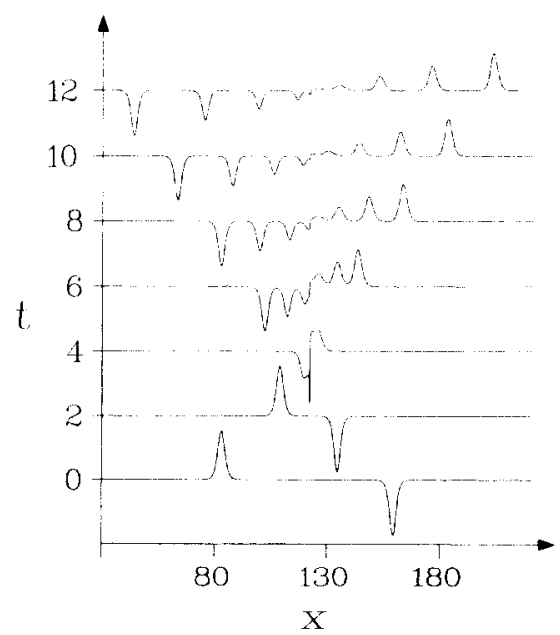

Fig. 1. Nonresonant collision of solitons with $p_{1}=0.48, p_{2}$ $=0.52\left(A_{1}=35.27, I_{\mathrm{li}}^{(1)}=146.9 ; A_{2}=-39.77, I_{\mathrm{Ii}}^{(2)}=\right.$ - 152.9); calculated with $h=0.05, \tau=0.025$. 
ton pairs in the case $p_{1}=0.48, p_{2}=0.52$. As can be clearly seen the outgoing solitons have suffered a negative time delay. For fixed $I_{\mathrm{Ii}}^{(1)}-I_{\mathrm{Ii}}^{(2)}$ the inelasticity is plotted in fig. 2 as a function of

$r=\left(I_{\mathrm{Ii}}^{(1)}+I_{\mathrm{Ii}}^{(2)}\right) /\left(I_{\mathrm{Ii}}^{(1)}-I_{\mathrm{Ii}}^{(2)}\right)$.

The inelasticity is indeed sharply peaked near, but not precisely on, the line $I_{\mathrm{Ii}}^{(1)}+I_{\mathrm{Ii}}^{(2)}=0$, although the maximum inelasticity moves closer to this line as $I_{\mathrm{Ii}}^{(1)}$ $-I_{\mathrm{Ii}}^{(2)}$ increases. The inelasticity can equally well be plotted in other directions in the $I_{\mathrm{Ii}}^{(1)} I_{\mathrm{Ii}}^{(2)}$ - plane. A sharp peak similar to that exhibited in fig. 2 is found if we vary the $p$ parameter of soliton 2 while keeping that of soliton 1 fixed. As an example, fig. 3a shows the inelasticity as a function of $I_{\mathrm{Ii}}^{(2)}$, or rather of $I_{\mathrm{li}}^{(1)}$ $+I_{\mathrm{Ii}}^{(2)}$, for $I_{\mathrm{Ii}}^{(1)}=26.67\left(p_{1}=0.40\right)$. Fig. 3a includes Santarelli's case $p_{1}=0.40, p_{2}=0.60$, which is marked in the figure. The peak shown would be even sharper if plotted as a function of $p_{2}$. For $p_{1}=0.40, \kappa_{\mathrm{II}}$ is greater than 0.30 only within the range $0.610<p_{2}$ $<0.624$. A much smoother variation of $\kappa_{\text {II }}$ is found along the line $I_{\mathrm{Ii}}^{(1)}+I_{\mathrm{Ii}}^{(2)}=0$ as is shown in fig. $3 \mathrm{~b}$. It is noteworthy that there appear to be no cusp-like features or discontinuities in figs. 2 and 3 corresponding to the onset of single or multiple pair production. For the resonant case $p_{1}=0.40, p_{2}=0.616$, which is at the peak of fig. $3 \mathrm{a}$, the collision process is shown in fig. 4. In contrast to Santarelli's case or that of fig. 1 the two solitons interact for a relatively long time before the various solitons emerge as noninteracting objects.

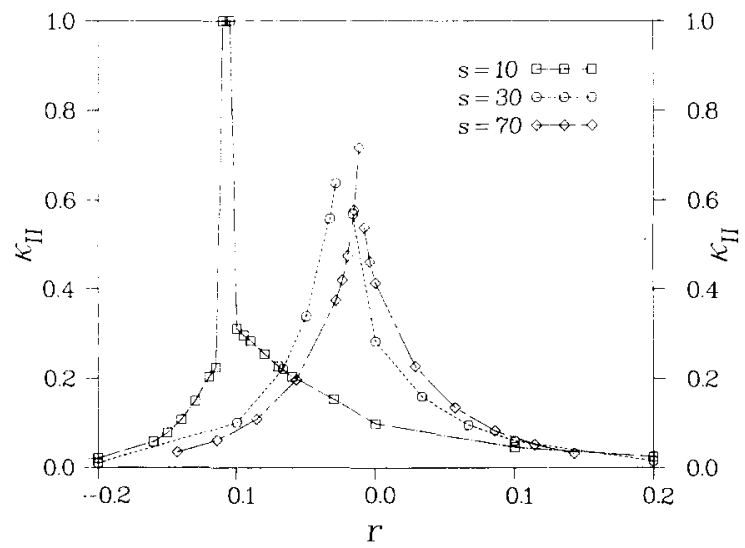

Fig. 2. The inelasticity $\kappa_{\mathrm{II}}$ as a function of $r=\left(I_{\mathrm{Ii}}^{(1)}+I_{\mathrm{Ii}}^{(2)}\right) /$ $\left(I_{\mathrm{Ii}}^{(1)}-I_{\mathrm{Ii}}^{(2)}\right)$ for fixed $s=I_{\mathrm{Ii}}^{(1)}-I_{\mathrm{Ii}}^{(2)}$. For $s=10$ there is no soliton production.

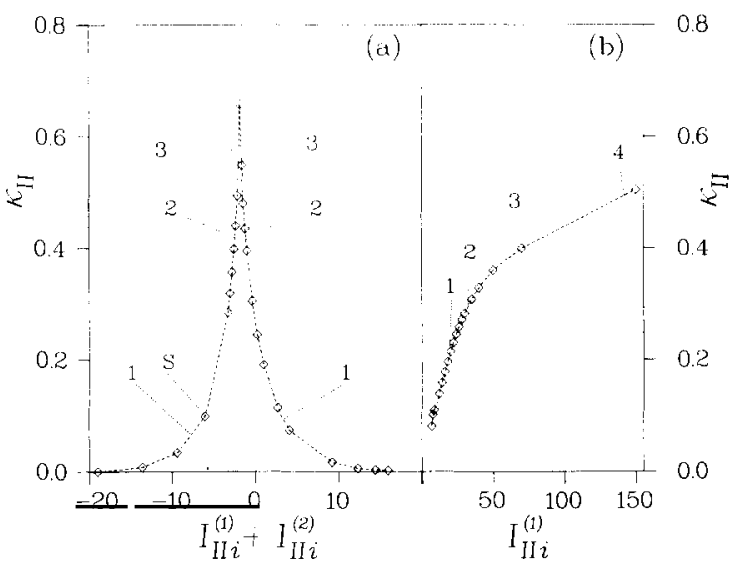

Fig. 3. (a) $\kappa_{\mathrm{II}}$ as a function of $I_{\mathrm{Ii}}^{(1)}+I_{\mathrm{Ii}}^{(2)}$ for fixed $p_{1}=0.40$. Santarelli's case is marked " $\mathrm{S}$ ". (b) $\kappa_{\mathrm{II}}$ as a function of $I_{\mathrm{Ii}}^{(1)}$ for $I_{\mathrm{Ii}}^{(1)}=-I_{\mathrm{li}}^{(2)}$. In both figures the approximate position of the onset of production of $1,2, \ldots$ pairs of solitons is indicated by the corresponding numeral.

Finally, fig. 5 shows the annihilating collision of two solitons, one with $I_{\mathrm{Ii}}^{(1)}=8.9\left(p_{1}=0.2659\right)$ and the other with $I_{\mathrm{Ii}}^{(2)}=-11.1\left(p_{2}=0.8386\right)$. Such collisions are presumably impossible for larger initial soliton areas $\left|I_{1 \mathrm{j}}\right|$ because an oscillatory wave without soliton character and of sufficient intensity to carry off the total value of $I_{\mathrm{II}}$ could not be produced.

The collision process merits some description.

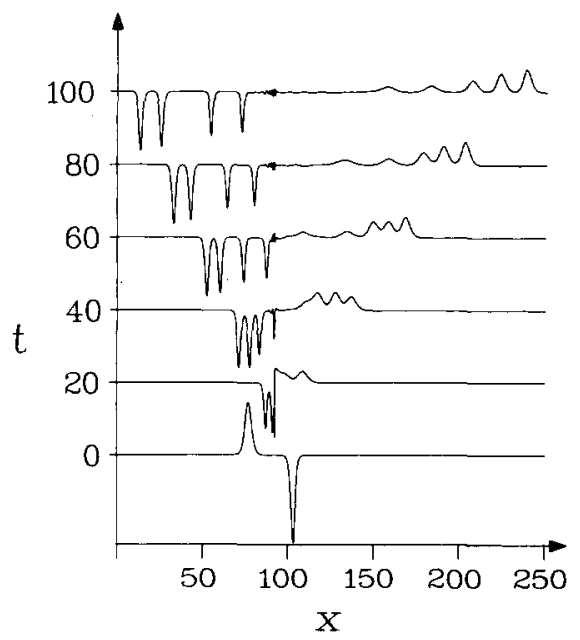

Fig. 4. Resonant collision of solitons with $p_{1}=0.40, p_{2}$ $=0.616\left(A_{1}=5.333, I_{\mathrm{Ii}}^{(1)}=26.667 ; A_{2}=-8.793, I_{\mathrm{Ii}}^{(2)}=\right.$ -28.550 ); calculated with $h=0.05, \tau=0.025$. 


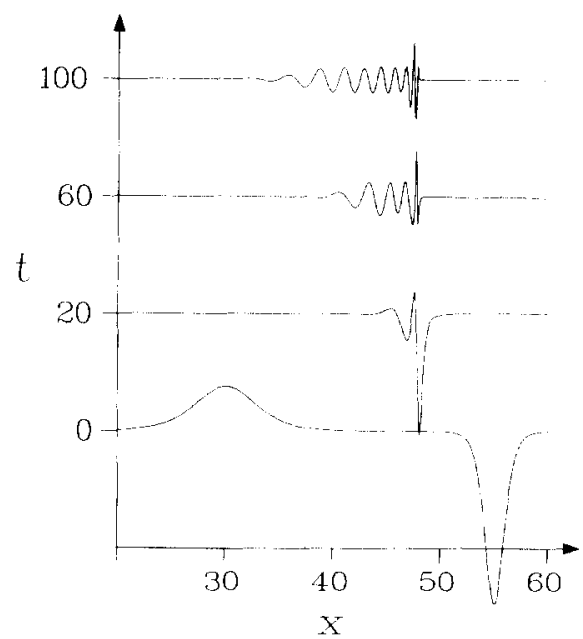

Fig. 5. Resonant, annihilating collision of solitons with $I_{\mathrm{Ii}}^{(1)}$ $=8.9, I_{\mathrm{Ii}}^{(1)}=-11.1\left(p_{1}=0.2659, A_{1}=1.183 ; p_{2}=0.8386\right.$, $A_{2}=-4.654$ ); calculated with $h=0.05, \tau=0.025$. The amplitudes in figs. 1,4 , and 5 are not to the same scale.

Whether or not the collision is substantially inelastic, the solitons come together to form a very localized rapidly varying large amplitude disturbance or "fireball', the $w w_{x}$ and $w_{x x t}$ terms of the RLW equation, eq. (1), become very large. This "fireball" then emits solitons until sufficiently diminished that it can no longer do so, and the remnant then emits a right-travelling oscillating wave. Except close to resonance the p-solitons and n-solitons emerge in a regular procession, in order of size; the p-solitons are usually less distinct in their nascent state than the n-solitons. It is a striking feature of the process that solitons are emitted in $\mathrm{p}, \mathrm{n}$-soliton pairs, although in certain cases described below it may be that the last soliton emission is of a lone soliton. In the emission process, each additional pair produced has the property that their total first invariant is small compared to the first invariants of each member of the pair. An $n$-soliton must have an amplitude greater than 3 in magnitude, as can be seen from eq. (4). The "fireball", when weak, either from a weak collision or following diminution by emission of solitons, will sometimes end by emitting an n-soliton-like object with amplitude less than 3 in magnitude. Such an object cannot be stable and in fact is not; after an initial quiescent phase it begins to oscillate in time, and sets up spatial oscillations. This can be seen in fig. 5. This unsuccessful attempt to create an n-soliton is usually accompanied by creation of a p-soliton-like object, though not in the case shown in fig. 5. We have been unable to decide whether these positive objects become p-solitons or not, as each maintains contact at its trailing edge with a long trough, and gradually decreases in amplitude, for as long as observed, which for some cases was 60 time units. Hence while there is a strong tendency for solitons to be emitted in $p, n$-soliton pairs, our data are equivocal as to whether or not this is strictly necessary. We have seen no instance, however, where the number of p-solitons and p-soliton-like objects emitted is less than the number of $n$-solitons, or exceeds that number by more than 1 .

Very near resonance the collision process changes in that $\mathrm{p}$-solitons and n-solitons are no longer emitted in order of their amplitudes, and the rightward-travelling disturbance breaks up only slowly, as seen in fig. 4 and as discussed above. In applying the inelasticity measure $\kappa_{\text {II }}$ we wait until the largest peaks have moved through the others and separated, and it is their amplitudes which we use to calculate $\kappa_{I I}$. However, although the assigning of an inelasticity is operationally well-defined even for a resonant collision, the usefulness of definition (5) is based on the notion that the original solitons preserve their identity in a collision, and that does not seem to be true at resonance. Indeed, for sufficiently "weak" collisions, such as those along the line $\frac{1}{2}\left(I_{\mathrm{Ii}}^{(1)}-I_{\mathrm{Ii}}^{(2)}\right)=10$ discussed above, the resonance condition corresponds to the annihilation of the incoming solitons, with no solitons of any sort being emitted.

In summary, we have shown that p-soliton-n-soliton collisions in the RLW equation can generate many p-soliton--n-soliton pairs, and that the inelasticity is a rapidly varying function of the soliton parameters. The most important parameter in this regard appears to be the signed area of the soliton, $I_{\mathrm{I} i \mathrm{i}}$. Although the RLW equation [8] has only been shown to be applicable to shallow-water for relatively weak waves $|w|$ $\lesssim 1$, and the weakest $n$-soliton has an amplitude of -3 , our work gives a further example of the subtlety and diversity of mathematical phenomena which may be found in the simplest of nonlinear wave equations. Our work suggests that it might be useful to examine other nonlinear wave equations which have soliton solutions to see whether their collisions also exibit resonance phenomena. 


\section{References}

[1] V.G. Makhankov, Phys. Rep. 35C (1978) 1.

[2] V.G. Makhankov, G. Kummer and A.B. Shvachka, preprint JINR P2-11913, Dubna (1978).

[3] Kh.O. Abdulloev, I.L. Bogolubsky and V.G. Makhankov, Phys. Lett. 56A (1976) 427.

[4] A.R. Santarelli, Nuovo Cimento 46B (1978) 179.

[5] Yu.A. Simonov and J.A. Tjon, Phys. Lett. 85B (1979) 380.
[6] J.C. Eilbeck and G.R. McGuire, J. Comp. Phys. 19 (1975) 43.

[7] J.C. Eilbeck and G.R. McGuire, J. Comp. Phys. 23 (1977) 63.

[8] T.B. Benjamin, J.L. Bona and J.J. Mahony, Phil. Trans. R. Soc. London 272A (1972) 47.

[9] P.D. Lax, in: Nonlinear evolution equations, ed. M.G. Crandall (Academic Press, New York, 1978) p. 207. 